\title{
DISTORTION OF A PHASE SPACE UNDER THE DARBOUX TRANSFORMATION"
}

\author{
Boris F. Samsonov \\ Tomsk State University, 36 Lenin Avenue, 634050 Tomsk, Russia,
}

The Darboux transformation operator technique is applied to construct exactly solvable anharmonic singular oscillator potentials and to study their coherent states. Classical system corresponding to a transformed quantum system is constructed with the help of the coherent states technique. It is shown that at classical level the Darboux transformation may be treated as a transformation of Kähler potential which leads to a distortion of the initial phase space.

PACS: 03.20.+i; 03.65.-w; 03.65.Fd; 03.65.Ge

\section{INTRODUCTION}

In quantum mechanics there exists a beautiful recipe to construct a family of exactly solvable Hamiltonians starting from an initial Hamiltonian $h_{0}$ for which the solutions of the Schrödinger equation $h_{0} \psi_{E}=E \psi_{E}$ are known. Since this method is very simple in applications and possesses an intrinsic charm it attracts a thorough attention of several generations of researches. A central point of this theory is a notion of a transformation operator introduced and investigated by Delsart and Lions.1 According to their approach, every operator $L$ intertwining the initial Hamiltonian $h_{0}$ and transformed Hamiltonian $h_{1}$,

$$
L h_{0}=h_{1} L
$$

is a transformation operator (originally called a transmutation operator 1 ). It is obvious that $L$ transforms solutions $\psi_{E}\left(\psi_{E} \notin \operatorname{ker} L\right)$ of the initial Schrödinger equation onto solutions $\varphi_{E}=L \psi_{E}$ of a transformed Schrödinger equation $h_{1} \varphi_{E}=E \varphi_{E}$

\footnotetext{
${ }^{a}$ To appear in J. Math. Phys. 1998, 39, No 1 or No 2.

${ }^{b}$ Electronic mail address: samsonov@phys.tsu.tomsk.su
} 
corresponding to the same eigenvalue $E$. In the case of a first order differential operator $L$ and a one dimensional Hamiltonian $h_{0}$, the relation (四) leads tol the transformation proposed by Moutard 3 and studied by Darboux 6 (see also Ref.5). If $L$ is a differential operator of order $N$ it is called目 $N$-order Darboux transformation operator. In this paper we will restrict ourselves by first order transformations.

The Darboux transformations operator technique is a very useful tool in obtaining and investigating new exactly solvable quantum problems. It enters many fields of physics and mathematics. In soliton theory the Darboux transformation permits one to construct and investigate properties of multisoliton solutions to nonlinear evolution equations (see Ref.6 and references therein). In quantum mechanics this technique takes the form of a factorization method. Using this method Schrödinger was able to solve the hydrogen atom problem in a pure algebraic manner. Subsequently, Infeld and Hall 8 generalized the Schrödinger method and obtained a wide class of solvable potentials. In the inverse quantum scattering problem 9 this transformation is used to construct potentials with given properties and to obtain appropriate solutions to the Schrödinger equation. New developments in this field have recently been presented in Ref.10. In the context of supersymmetric quantum mechanics (for reviews see Ref.11) the Hamiltonian $h_{0}$ and its superpartner $h_{1}$ form a matrix-valued Hamiltonian (so called super Hamiltonian) $\mathcal{H}$. Darboux transformation operator $L$ and its Hermitian conjugate $L^{+}$participate in construction of supercharge operators which, together with $\mathcal{H}$, form a superalgebra. Initially the supersymmetric quantum mechanics served as a toy model to illustrate the problem of supersymmetry breaking in quantum field theories. Recently, new features in s upersymmetry breakdown in quantum mechanics caused by higher order Darboux transformations have been discovered.12 Besides quantum field theories the same ideas find applications in many others fields of theoretical physics such as nuclear, a tomic, solid state and statistical physics.11

In spite of the long period of application of the Darboux transformation operator method to quantum mechanical problems the question of correspondence between the transformed quantum system and a classical system has not been properly discussed in the literature. In a recent paper 13 a classical mechanics counterpart of the transformed system has been described. It consists in addition of a total time 
derivative of a purely imaginary function to the Lagrangian of a classical system. This transformation does not affect the equations of motion. Canonical quantization of the new classical system gives the transformed quantum system.

The concept of coherent states plays an important role in several fields of physics and mathematics (see, for example, Refs.14-15). In this paper we exploit the property of coherent states to establish the correspondence between the quantum mechanics and the classical mechanics. For any quantum system we can construct using the coherent states technique14, 16 a classical system possessing the following remarkable property. The geometric quantization 17 of the resulting system gives holomorphic representation of the initial quantum system. By these means we may establish a correspondence between quantum and classical systems. Recently it has been noted 18 that application of the Darboux transformation operator to the coherent states of the initial quantum system gives a set of states which may be treated as coherent states of the transformed system. This observation open s the door to establishing a correspondence between transformed quantum and classical systems and obtaining a classical counterpart of the Darboux transformation.

In this paper we realize the above program for a system with the Hamiltonian

$$
h_{0}=-d^{2} / d x^{2}+x^{2} / 4+b / x^{2}, \quad b>0, \quad x \in[0, \infty) .
$$

We will not discuss the significance of the spectral problem for the Hamiltonian (22) (more precisely, for its closure in the Hilbert space $H$ of square integrable on interval $[0, \infty)$ functions with the zero boundary condition at the origin). We only notice the fact that this Hamiltonian appears in different physical problems such as central potential problem, Calogero problem 19, fractional statistics and anyons 20, two-dimensional QCD21, and others.

We obtain exactly solvable partners for the Hamiltonian (21). Next we construct the coherent states for the transformed a lá Darboux Hamiltonians. These states as well as the coherent states for the input system (initial coherent states) are labeled by the points of a unite disk on the complex $z$-plane. We consider two different Kähler structures on the unit disk. The first one is the well known Poincaré model of the Lobachevsky plane and the second is the Darboux transformation of the first. The expression for the transformed Kähler potential is obtained. We also notice 
Boris F. Samsonov: Distortion of a phase space

that in contrast to the initial manifold the transformed one has a varying curvature. The integration measure realizing the identity decomposition for the transformed coherent states is calculated. The holomorphic representation for the discrete basis set as well as for the coherent states, lowering and raising operators and Darboux transformation operators is constructed. It is established that at the classical level the Darboux transformation may be considered as such a transformation of the Kähler potential and Hamilton function that preserves the equations of motion.

\section{COHERENT STATES OF SINGULAR OSCILLATOR}

In this section we briefly review the well known 14 properties of the coherent states for the Hamiltonian (2) that we will need further.

The dynamical symmetry algebra for the quantum system with the Hamiltonian (2) is $s u(1,1)$. In coordinate representation the generators of this algebra are expressed in terms of the harmonic oscillator annihilation $a$ and creation $a^{+}$operators

$$
\begin{gathered}
k_{0}=\frac{1}{2} h_{0}, \quad k_{+}=\frac{1}{2}\left[\left(a^{+}\right)^{2}-b / x^{2}\right], \quad k_{-}=\frac{1}{2}\left[a^{2}-b / x^{2}\right], \\
a=d / d x+x / 2, \quad a^{+}=-d / d x+x / 2 .
\end{gathered}
$$

and satisfy the standard commutation relations

$$
\left[k_{0}, k_{ \pm}\right]= \pm k_{ \pm}, \quad\left[k_{-}, k_{+}\right]=2 k_{0}
$$

Since we consider an irreducible representation the corresponding $s u(1,1)$ Casimir operator takes the constant value $\mathcal{C}=\frac{1}{2}\left[k_{+} k_{-}+k_{-} k_{+}\right]-k_{0}^{2}=3 / 16-b / 4=k(1-k)$. The value of $k=1 / 2+(1 / 4) \sqrt{1+4 b}$ defines the ground state (vacuum) energy $E_{0}=2 k$. The vacuum state $|0\rangle$ is defined by the equations: $k_{-}|0\rangle=0$ and $k_{0}|0\rangle=k|0\rangle$. Other discrete basis eigenfunctions $|n\rangle$ of the Hamiltonian $h_{0}$ are defined by means of the raising operator $k_{+}$

$$
\begin{gathered}
k_{+}|n\rangle=-\sqrt{(n+1)(n+2 k)}|n+1\rangle, \\
|n\rangle=(-1)^{n} \sqrt{\frac{\Gamma(2 k)}{n ! \Gamma(2 k+n)}}\left(k_{+}\right)^{n}|0\rangle, \quad k_{0}|n\rangle=(k+n)|n\rangle
\end{gathered}
$$

and in the coordinate representation they have the form

$$
\psi_{n}(x)=\langle x \mid n\rangle=\left[n ! 2^{1-2 k} \Gamma^{-1}(n+2 k)\right]^{1 / 2} x^{2 k-1 / 2} \exp \left(-x^{2} / 4\right) L_{n}^{2 k-1}\left(x^{2} / 2\right),
$$


where $L_{n}^{\alpha}(z)$ are the Laguerre polynomials.

The coherent states $|z\rangle$ can be obtained by applying of the unitary displacement operator $D_{z}$ to the vacuum state

$$
\begin{gathered}
|z\rangle=D_{z}|0\rangle=e^{z k_{+}} \exp \left[\ln (1-z \bar{z}) k_{0}\right] e^{-\bar{z} k_{-}}|0\rangle=N_{0 z} \sum_{n=0}^{\infty} a_{n} z^{n}|n\rangle, \\
N_{0 z}=(1-z \bar{z})^{k}, \quad a_{n}=(-1)^{n} \sqrt{\frac{\Gamma(2 k+n)}{n ! \Gamma(2 k)}}, \quad|z|<1 .
\end{gathered}
$$

In the coordinate representation they look like

$$
\begin{gathered}
\psi_{z}(x)=2^{1 / 2-k} \Gamma^{-1 / 2}(2 k)(1-z)^{-2 k}(1-z \bar{z})^{k} x^{2 k-1 / 2} \times \\
\exp \left[-(1 / 4)(1-z)^{-1}(1+z) x^{2}\right], \quad|z|<1 .
\end{gathered}
$$

The linear manifold spanned by the vectors $\{|z\rangle\}$ forms an everywhere dense set in the Hilbert space $H$ with the following identity decomposition:

$$
\int_{|z|<1}|z\rangle\langle z| d \mu(z)=1, \quad d \mu(z)=\frac{2 k-1}{\pi}(1-z \bar{z})^{-2} d z d \bar{z} .
$$

The system $\{|z\rangle\}$ is overcomlete. The completeness of different subsystems of this system was studied in detail in Ref.22.

The Fourier coefficients $c_{n}$ of a vector $|\psi\rangle \in H$ with respect to the basis $\{|n\rangle\}$ define a holomorphic representation $\psi(z)$ of the vector $|\psi\rangle$ in the space of functions which are holomorphic in the unit disk

$$
\langle\bar{z} \mid \psi\rangle=N_{0 z} \psi(z), \quad \psi(z)=\sum_{n=0}^{\infty} a_{n} c_{n} z^{n} .
$$

The functions $\psi_{n}(z)=a_{n} z^{n}$ realize the holomorphic representation of the basis vectors $|n\rangle$. For the holomorphic representation of the coherent state vectors we have

$$
\zeta^{(0)}(z)=N_{0 z}^{-1}\langle\bar{z} \mid \zeta\rangle=(1-\zeta \bar{\zeta})^{k}(1-\zeta z)^{-2 k}
$$

Let $\mathcal{L}$ be the lineal of entire analytic functions $\psi(z)$ defined in the unit disk such that

$$
\int_{|z|<1}|\psi(z)|^{2}\left(1-|z|^{2}\right)^{2 k} d \mu(z)<\infty .
$$

If now we define an inner product in the lineal $\mathcal{L}$

$$
\left\langle\psi_{1}(z) \mid \psi_{2}(z)\right\rangle \equiv \int_{|z|<1} e^{-f^{(0)}} \bar{\psi}_{1}(z) \psi_{2}(z) d \mu(z)=\left\langle\psi_{1} \mid \psi_{2}\right\rangle,
$$


where $\left\langle\psi_{1} \mid \psi_{2}\right\rangle$ is the inner product in the Hilbert space $H$ then the completion of $\mathcal{L}$ with respect to this inner product gives the Hilbert space $\mathcal{H}$. The function $f^{(0)}=\ln |\langle 0 \mid z\rangle|^{-2}=\ln (1-z \bar{z})^{-2 k}$ is the Kähler potential in the unit disk. The corresponding metric has the form

$$
d s^{2}=g d z d \bar{z}, \quad g=\frac{\partial^{2} f^{(0)}}{\partial z \partial \bar{z}}=\frac{2 k}{(1-z \bar{z})^{2}} .
$$

As usually the imaginary part of this metric defines a symplectic 2-form $\omega=$ $-i g d z \wedge d \bar{z}$ and consequently a Poisson bracket $\left\{H_{1}, H_{2}\right\}$ of the functions $H_{1}$ and $H_{2}$ defined on the unit disk. The unit disk represents now a phase space of a constant Gauss curvature $\mathcal{K}^{(0)}=-\frac{2}{k}$. Making use of the covariant Berezin symbols23 we establish a one-to-one correspondence between the classical observables and the holomorphic representation of the quantum mechanical operators. For example, the following classical observables:

$$
\begin{gathered}
K_{0}=\left\langle\bar{z}\left|k_{0}\right| \bar{z}\right\rangle=k \frac{1+z \bar{z}}{1-z \bar{z}}, \\
K_{-}=\left\langle\bar{z}\left|k_{-}\right| \bar{z}\right\rangle=\frac{2 k \bar{z}}{1-z \bar{z}}, \quad K_{+}=\left\langle\bar{z}\left|k_{+}\right| \bar{z}\right\rangle=\frac{2 k z}{1-z \bar{z}}
\end{gathered}
$$

correspond to the generators of $s u(1,1)$ algebra of quantum operators. They form the basis of the algebra isomorphic to $s u(1,1)$ with respect to the introduced Poisson bracket.

If we choose $K_{0}$ as a classical Hamilton function then the evolution of the system is described by the Hamilton equations

$$
\dot{z}=\left\{z, K_{0}\right\}=-i z, \quad \dot{\bar{z}}=\left\{\bar{z}, K_{0}\right\}=i \bar{z},
$$

where the dot stands for the time derivative.

Geometric quantization 17 of the obtained classical system[leads to the holomorphic representation of the quantum system described above. The value $h=(2 k)^{-1}$ plays the role of the Plank constant.14

To conclude this section we present the explicit expressions for the Bergman kernel which takes off the integration in the $\mathcal{L}$-space

$$
\delta^{(0)}\left(z, \bar{z}^{\prime}\right)=\sum_{n=0}^{\infty} \psi_{n}(z) \bar{\psi}_{n}\left(z^{\prime}\right)=\left(1-z \bar{z}^{\prime}\right)^{-2 k},
$$

${ }^{c}$ In our case this is a quantization on the Lobachevsky pland 14 which really is a Berezin 24 quantization on a Kähler manifold 
and for the $s u(1,1)$ generators

$$
k_{0}(z)=z \frac{d}{d z}+k, \quad k_{+}(z)=z^{2} \frac{d}{d z}+2 k z, \quad k_{-}(z)=\frac{d}{d z} .
$$

in holomorphic representation.

\section{DARBOUX TRANSFORMATION OF COHERENT STATES}

In a simplest case 2 the Darboux transformation operator is the first order differential operator of the form

$$
L=-L_{0}(x)+d / d x=-u^{\prime}(x) / u(x)+d / d x
$$

where the prime denotes the derivative with respect to $x$. When acting on the solutions $\psi_{n}(x)$ of the initial Schrödinger equation

$$
h_{0} \psi_{n}(x)=E_{n} \psi_{n}(x), \quad E_{n}=2 n+2 k
$$

it transforms them into the solutions of another Schrödinger equation

$$
\varphi_{n}(x)=N_{n} L \psi_{n}(x), \quad h_{1} \varphi_{n}(x)=E_{n} \varphi_{n}(x)
$$

with the same eigenvalues $E_{n}$. The factor $N_{n}$ is introduced to ensure that the states $\varphi_{n}$ are normalized to unity. New exactly solvable Hamiltonian $h_{1}=h_{0}+A(x)$ is defined by the potential difference $A=A(x)=-2(\ln u)^{\prime \prime}$. The function $u=u(x)$ called transformation function国 is a solution to the initial Schrödinger equation

$$
h_{0} u(x)=\alpha u(x)
$$

with $\alpha \leqslant E_{0}$.

For the sake of simplicity we will restrict ourselves to the case when $\alpha<E_{0}$, $u(x) \neq 0 \forall x \in(0, \infty)$ and $1 / u(x)$ is not a square integrable function in the interval $[0, \infty)$. In this case $u \notin H$ and the set $\left\{\left|\varphi_{n}\right\rangle\right\}$ constitutes a complete basis in the Hilbert space $H$ provided the initial system $\left\{\left|\psi_{n}\right\rangle\right\}$ is complete.2 In terms of the supersymmetric quantum mechanics this case corresponds to a broken supersymmetry. 
Let us now choose the following solution of the initial equation (8):

$$
\begin{gathered}
u=u_{p}(x)=x^{2 k-1 / 2} L_{p}^{2 k-1}(y) \exp \left(x^{2} / 4\right), \quad y=-x^{2} / 2 \\
\alpha=-2(k+p), \quad p=0,1,2, \ldots
\end{gathered}
$$

as the transformation function. It is worth stressing that $L_{p}^{2 k-1}(y) \neq 0 \forall x \neq 0$ when $y=-x^{2} / 2(<0)$ and, hence, the function $u_{p}(x)$ is nodeless for $x>0$. The function $L_{0}(x)=L_{0 p}(x)$ has in this case the form

$$
L_{0 p}(x)=\frac{1-4 k}{2 x}-\frac{x}{2}-\frac{x L_{p-1}^{2 k}(y)}{L_{p}^{2 k-1}(y)}
$$

and for the potential difference we obtain

$$
\begin{gathered}
A=A_{p}(x)=-1+\frac{4 k-1}{x^{2}}- \\
2 \frac{x^{2} L_{p-2}^{2 k+1}(y)+L_{p-1}^{2 k}(y)}{L_{p}^{2 k-1}(y)}+2 x^{2}\left[\frac{L_{p-1}^{2 k}(y)}{L_{p}^{2 k-1}(y)}\right]^{2}, \quad y=-x^{2} / 2 .
\end{gathered}
$$

It is worth noticing that because of the condition $L_{p}^{2 k-1}(y) \neq 0$ for $x \neq 0$ the potential difference $A_{p}(x)$ has a single pole at $x=0$. The asymptotic behavior of this function near the point $x=0$ is as follows: $A_{p}(x) \rightarrow-1-p / k+(4 k-1) / x^{2}$. In the limit as $x \rightarrow \infty$ this function tends to -1 . For $b \neq 0$ the behavior of the transformed potentials $V_{p}(x)=V_{0}(x)+A_{p}(x)$ is nearly the same as the behavior of the initial potential $V_{0}(x)=x^{2} / 4+b / x^{2}$. There is only a small difference in the region of the minimum. When $b=0$ the initial potential is regular at $x=0$ whereas all $V_{p}(x)$ are singular: $V_{p}(x) \rightarrow-1-4 p / 3+2 / x^{2}$. As an illustration, we give the explicit expression for two first potential differences

$$
A_{0}(x)=-1+\frac{4 k-1}{x^{2}}, \quad A_{1}(x)=A_{0}(x)+\frac{4}{4 k+x^{2}}-\frac{32 k}{\left(4 k+x^{2}\right)^{2}} .
$$

Thus, the transformed Hamiltonians $h_{1}=h_{1}^{(p)}=-d^{2} / d x^{2}+V_{p}(x)$ are bounded from below and are essentially self adjoint operators defined on the dense set in the Hilbert space $H$. All these Hamiltonians have only discrete spectrum. The position of the energy levels is the same as before transformation: $E_{n}=2 n+2 k, n=0,1,2, \ldots$.

The operator $L^{+}=-L_{0}(x)-d / d x$ realizes the transformation in the inverse direction

$$
\left|\psi_{n}\right\rangle=N_{n} L^{+}\left|\varphi_{n}\right\rangle
$$


and together with $L$ participates in the following factorization:

$$
L^{+} L=h_{0}-\alpha, \quad L L^{+}=h_{1}-\alpha
$$

We note that in our case operators $L$ and $L^{+}$are well-defined $\forall \psi \in H$ and are conjugated to each other with respect to the inner product in the space $H$. This observation together with the factorization properties (10) is very useful in calculating integrals involving transformed functions. In particular, for the normalization constant $N_{n}$ we readily find $N_{n}^{-2}=\left\langle\varphi_{n}\left|h_{1}-\alpha\right| \varphi_{n}\right\rangle=E_{n}-\alpha=2 p+4 k+2 n$.

Now, by following the method of Ref.18 we find the transformed coherent states

$$
\begin{gathered}
\varphi_{z}(x)=N_{1 z} L \psi_{z}(x)=N \sum_{n=0}^{\infty} b_{n} z^{n} \varphi_{n}, \\
N=N_{0 z} N_{1 z} / N_{0}, \quad b_{n}=a_{n} N_{0} / N_{n}
\end{gathered}
$$

From the factorization properties (10) one readily derives the value of the normalization constant

$$
N_{1 z}^{-2}=\frac{4 k+2 p-2 p z \bar{z}}{1-z \bar{z}}
$$

Using the identity operator

$$
\sum_{n=0}^{\infty}\left|\varphi_{n}\right\rangle\left\langle\varphi_{n}\right|=1
$$

one can calculate the integration measure $\nu(z)$ which realizes the following identity decomposition:

$$
\int_{|z|<1}\left|\varphi_{z}\right\rangle\left\langle\varphi_{z}\right| d \nu(z)=1
$$

For this purpose we pass from the complex variables $z$ and $\bar{z}$ to the polar ones $z=$ $\sqrt{x} \exp i \phi$ and look for the measure in the form $d \nu=\frac{1}{2} h(x) d x d \phi$. The integration over the angle variable $\phi$ in (12) is trivial. Then making use of the formula (11), we obtain the equation for the function $h(x)$

$$
\pi \int_{0}^{1} d x h(x) \frac{x^{n}(1-x)^{2 k+1}}{2 k+p-p x}=\frac{n ! \Gamma(2 k)}{\Gamma(n+2 k)(n+2 k+p)} .
$$

So, we arrive to a moments problem well known in mathematics.25 In our case the solution to this problem can readily be obtained with the help of the integral representation of the beta-function

$$
B(a, b)=\frac{\Gamma(a) \Gamma(b)}{\Gamma(a+b)}=\int_{0}^{1} x^{a-1}(1-x)^{b-1} d x
$$


and the following identity:

$$
\frac{\Gamma(n+1) \Gamma(2 k)}{(n+2 k+p) \Gamma(n+2 k)}=\sum_{j=0}^{p} C_{p}^{j} \frac{2 k-1}{2 k+p-j-1} B(n+j-1,2 k+p-j)
$$

where $C_{p}^{j}$ are the binomial coefficients. It is not difficult to prove this identity by induction. The final expression for the function $h(x)$ is

$$
h(x)=\frac{2 k-1}{\pi}(2 k+p-p x) \sum_{j=0}^{p} C_{p}^{j} \frac{x^{j}(1-x)^{p-j-2}}{2 k+p-j-1} .
$$

Once we know the measure $\nu(z)$ we can construct the holomorphic representation $\varphi(z)$ in the unit disk of a vector $|\varphi\rangle \in H$ which is defined by its Fourier coefficients $c_{n}$ with respect to the base $\left\{\left|\varphi_{n}\right\rangle\right\}$

$$
\left\langle\varphi_{\bar{z}} \mid \varphi\right\rangle=N \varphi(z), \quad \varphi(z)=\sum_{n=0}^{\infty} b_{n} c_{n} z^{n} .
$$

It follows from these relations the holomorphic representation for the transformed discrete basis vectors $\varphi_{n}(z)=b_{n} z^{n}$ and for the transformed coherent states

$$
\zeta^{(1)}(z)=N^{-1}\left\langle\varphi_{\bar{z}} \mid \varphi_{\zeta}\right\rangle=\frac{2 k+p+p \zeta z}{\sqrt{2 k+p}} \frac{(1-\zeta \bar{\zeta})^{k+1 / 2}}{(1-\zeta z)^{2 k+1}}(2 k+p-p \zeta \bar{\zeta})^{-1 / 2} .
$$

With the help of the transformation operators $L$ and $L^{+}$we construct the lowering and raising operators $p_{ \pm}=L k_{ \pm} L^{+}$for the transformed discrete basis functions

$$
\begin{gathered}
p_{+}\left|\varphi_{n}\right\rangle=-\left(N_{n} N_{n+1}\right)^{-1}[(n+1)(n+2 k)]^{1 / 2}\left|\varphi_{n+1}\right\rangle, \\
p_{-}\left|\varphi_{n}\right\rangle=-\left(N_{n} N_{n-1}\right)^{-1}[n(n+2 k-1)]^{1 / 2}\left|\varphi_{n-1}\right\rangle .
\end{gathered}
$$

Together with the operator $p_{0}=\frac{1}{2} h_{1}$ they form a non-linear algebra

$$
\left[p_{0}, p_{ \pm}\right]= \pm p_{ \pm}, \quad\left[p_{-}, p_{+}\right]=2\left(2 k(1-k)-p_{0} \alpha+4 p_{0}^{2}\right)\left(2 p_{0}-\alpha\right)
$$

It is interesting that the operators $p_{ \pm}$are of the fourth degree in derivative in the coordinate representation while in the holomorphic one they are of the second degree

$$
\begin{gathered}
p_{0}(z)=z \frac{d}{d z}+k, \quad p_{-}(z)=2 z \frac{d^{2}}{d z^{2}}+2(2 k+p) \frac{d}{d z}, \\
p_{+}(z)=2 z^{3} \frac{d^{2}}{d z^{2}}+2 z(2 k+p+2)\left(z \frac{d}{d z}+2 k\right) .
\end{gathered}
$$


Since the operators $L$ and $L^{+}$realize the correspondence between the basis sets $\left\{\left|\psi_{n}\right\rangle\right\}$ and $\left\{\left|\varphi_{n}\right\rangle\right\}$ we can find their holomorphic representation

$$
L(z)=\sqrt{\frac{2}{2 k+p}}\left(z \frac{d}{d z}+2 k+p\right), \quad L^{+}(z)=\sqrt{2(2 k+p)} .
$$

By assuming that the inner product of two vectors $\left|\varphi_{1}\right\rangle$ and $\left|\varphi_{2}\right\rangle$ is independent on the representation used and taking into account the identity decomposition (12) we can equip the lineal $\mathcal{L}$ with a new inner product

$$
\left\langle\varphi_{1} \mid \varphi_{2}\right\rangle=\left\langle\varphi_{1}(z) \mid \varphi_{2}(z)\right\rangle=\int_{|z|<1} \bar{\varphi}_{1}(z) \varphi_{2}(z) e^{-f^{(1)}} d \nu(z) .
$$

It is naturally to declare the function

$$
f^{(1)}=f^{(1)}(z, \bar{z})=\ln \left|\left\langle\varphi_{0} \mid \varphi_{z}\right\rangle\right|^{-2}=f^{(0)}+\ln \frac{\left\langle\psi_{z}\left|h_{0}-\alpha\right| \psi_{z}\right\rangle}{E_{0}-\alpha}
$$

a new Kähler potential. It then follows the expression for a new Hermit metric

$$
g^{(1)}=f_{z \bar{z}}^{(1)}=\frac{2 k+1}{(1-z \bar{z})^{2}}-\frac{p(2 k+p)}{(2 k+p-p z \bar{z})^{2}} .
$$

The unit disk represents now a phase space with the varying Gauss curvature $\mathcal{K}^{(1)}=-\frac{2}{g} \frac{\partial^{2}}{\partial z \partial \bar{z}} \ln g^{(1)}$. The explicit expression for $\mathcal{K}^{(1)}$ is rather complicated so that be omitted here. We will note only that $\mathcal{K}^{(1)} \rightarrow 0$ when $k \rightarrow \infty$.

The function

$$
\delta^{(1)}\left(z, \bar{z}^{\prime}\right)=\sum_{n=0}^{\infty} \varphi_{n}(z) \bar{\varphi}_{n}\left(z^{\prime}\right)=\left(1-z \bar{z}^{\prime}\right)^{-2 k-1} \frac{2 k+p-p z \bar{z}}{2 k+p}
$$

plays the role of the transformed Bergman kernel which takes off the integration with respect to the new inner product (13).

By making use of the covariant Berezin symbols we can establish correspondence between the quantum mechanical operators and the classical observables which are the functions of a point in the unit disk. The function

$$
H_{1}=\left\langle\varphi_{\bar{z}}\left|h_{1}\right| \varphi_{\bar{z}}\right\rangle=2 k+\frac{4 k z \bar{z}(2 k+p+1-p z \bar{z})}{(1-z \bar{z})(2 k+p-p z \bar{z})}
$$

corresponds to the transformed Hamiltonian operator and therefore it is the transformed Hamilton function. Similarly, the functions $P_{+}$and $P_{-}$,

$$
P_{+}=\bar{P}_{-}=\left\langle\varphi_{\bar{z}}\left|p_{+}\right| \varphi_{\bar{z}}\right\rangle=
$$




$$
\frac{4 k \bar{z}}{2 k+p-p z \bar{z}}\left[\frac{(2 k+1)(2 k+2)}{(1-z \bar{z})^{2}}+(p-1) \frac{2 k+1}{1-z \bar{z}}+p(p-1)\right],
$$

correspond to the lowering and raising operators.

The change of the Kähler potential induces the change of the symplectic form which remains closed because of its dimension. As a consequence we have another Poisson brackets in the unit disk. The functions $P_{0}=\frac{1}{2} H_{1}$ and $P_{ \pm}$form now neither a closed Lie algebra nor a polynomial one with respect to the new Poisson bracket but it is not difficult to check the following relations:

$$
\left\{P_{0}, P_{ \pm}\right\}= \pm i P_{ \pm}, \quad\left\{P_{0}, z\right\}=i z, \quad\left\{P_{0}, \bar{z}\right\}=-i \bar{z}
$$

Two latter relations mean that the equation of trajectories for the transformed classical system in the transformed phase space remains unchanged under the Darboux transformation.

\section{CONCLUSION}

Using the coherent states technique we have constructed a classical counterpart of the transformed a lá Darboux quantum singular oscillator. It has been shown that the Darboux transformation for this system translates into the transformation of the Kähler potential. New Kähler potential has a supplementary summand of the form $\ln \left(H_{0}-\alpha\right)$ where $H_{0}$ is initial Hamilton function corresponding to the quantum Hamiltonian operator for the singular oscillator. This leads to the distortion of the initial phase space. However, the distortion is consistent with the change of the Hamilton function in such a manner that the curves of a constant energy (classical trajectories) in the transformed phase space remain unchanged. A similar observation has recently been discusse $d$ in Lagrangian formalism and for the canonical method of quantization.13

\section{ACKNOWLEDGMENT}

This research has been partially supported by RFBR grant No 97-02-16279. 
Boris F. Samsonov: Distortion of a phase space

1 J. Delsart, Comp. Rend. Acad. Sci. Paris. 206. 178 (1938); Colloque Int. Nancy. 29 (1956); J. L. Lions, Colloques Intern. Nancy. 125 (1956); J. Delsart and J. L. Lions, Comment. Math. Helv. 32. 113 (1957).

2 V. G. Bagrov and B. F. Samsonov, Fiz. Elem. Chastits Atom. Yadra 28. 951 (1997); Teor. Math. Phys. 104. 1051 (1995).

3 T. F. Moutard, J. École Polytech. XLV. 1 (1878).

${ }^{4}$ G. Darboux, Compt. Rend. Acad. Sci. Paris. 94. 1343 (1882); 94. 1456 (1882); Leçons sur la théorie générale des surfaces et les application géométriques du calcul infinitésimale. Deuxiem partie. Paris, Gautier-Villar et Fils (1889).

5 E. L. Ince, Ordinary differential equations, New York, Dover (1926).

6 V. Matveev and M. Salle, Darboux transformations and solitons, Berlin, Springer (1991);

7 E. Schrödinger, Proc. Roy. Irish. Acad. A . 46. 9 (1940); 47. 53 (1941); 47183 (1941).

8 T. E. Infeld, Phys. Rev. 59. 737 (1941); H. Hull and T. E. Infeld, Phys. Rev. 74. 905 (1948); T. E. Infeld and H. Hull, Rev. Mod. Phys. 53. 21 (1951).

9 L. D. Faddev, Usp. Mat. Nauk 14. 57 (1959); B. N. Zakhariev and A. A. Suzko, Direct and inverse problems. Springer-Verlag, Heidelberg, 1990.

10 A. A. Suzko, Int. J. Mod. Phys. A. 12. 277 (1997); B. F. Samsonov, J. Phys. A: Math. Gen. 28. 6989 (1995).

11 G. Junker, Supersymmetric methods in quantum and statistical physics. Springer, Berlin (1996); F. Cooper, A. Khare and U. Sukhatme, Phys. Rep. 251. 267 (1995); V. A. Kostelecky and D. K. Campbell, Supersymmetry in physics. North-Holla nd, Amsterdam (1985).

12 B. F. Samsonov, Mod. Phys. Lett. A. 11.1563 (1996).

13 A. A. Neto and E. D. Filho, Mod. Phys. Lett. A. 12. 899 (1997).

14 A. M. Perelomov, Generalized Coherent States and Their Applications. Springer, Berlin (1986).

15 I. A. Malkin and V. I. Man'ko, Dynamical Symmetries and Coherent States of Quantum Systems, Moscow, Nauka (1979); J. R. Klauder and B. S. Skagerstam, Coherent states: applications in physics and mathematical physics. Singapore, World Scientific (1985).

16 J. R. Klauder, J. Math. Phys. 4, 1055 (1963); 4, 1058 (1963); 5, 177 (1964).

17 B. Kostant, Lect. Not. Math. 170, 87 (1970); A. Kirillov, Eléments de la Théorie des Représentation, Moscou, Mir (1974); N. M. J. Woodhouse, Geometric quantization. Oxford (1991). J. R. Klauder, Geometric quantization from a coherent state viewpoint, E-print quant-ph/9510008 (1995).

18 V. G. Bagrov and B. F. Samsonov, Zh. Éksp. Teor. Fiz. 109, 1105 (1996); J. Phys. A 29, 1011 (1996).

19 F. Calogero, J. Math. Phys. 10, 2191 (1969); 10, 2197 (1969); 12, 419 (1971).

20 J. M. Leinaas and J. Mirheim, Phys. Rev. B 37, 9286 (1988); A. P. Polychronacos, Nucl. Phys. B 324, 597 (1989); F. D. M. Haldane, Phys. Rev. Lett. 67, 937 (1991). 
21 J. A. Minahan and A. P. Polychronacos, Phys. Lett. B 326, 288 (1994).

22 A. M. Perelomov, Funct. Analis. Appl. 7, 57 (1973).

23 F. A. Berezin, Izv. Akad. Nauk SSSR, ser. Mat. 37, 1134 (1972).

24 F. A. Berezin, Dokl. Akad. Nauk SSSR, ser. Mat. 211. 1263 (1973); Izv. Akad. Nauk SSSR, ser. Mat. 38. 1116 (1974); 39. 363 (1975).

25 N. I. Akhiezer, Classical moments problem. Moscow (1961). 